\title{
Lumen
}

Selected Proceedings from the Canadian Society for Eighteenth-Century Studies

\section{Mirabeau sous le sceau du secret : l'écriture épistolaire à l'épreuve de la surveillance pénitentiaire}

\section{Sophie Rothé}

Volume 37, 2018

URI : https://id.erudit.org/iderudit/1042228ar

DOI : https://doi.org/10.7202/1042228ar

Aller au sommaire du numéro

Éditeur(s)

Canadian Society for Eighteenth-Century Studies / Société canadienne d'étude du dix-huitième siècle

ISSN

1209-3696 (imprimé)

1927-8284 (numérique)

Découvrir la revue

Citer cet article

Rothé, S. (2018). Mirabeau sous le sceau du secret : l'écriture épistolaire à

l'épreuve de la surveillance pénitentiaire. Lumen, 37, 135-150.

https://doi.org/10.7202/1042228ar 


\title{
Mirabeau sous le sceau du secret: l'écriture épistolaire à l'épreuve de la surveillance pénitentiaire
}

\author{
Sophie Rothé \\ Université de Tours
}

«Chaque siècle a son esprit qui le caractérise; l'esprit du nôtre semble être celui de la libertél », écrivait Diderot. Dans cet esprit, les écrits carcéraux du comte de Mirabeau, qui fut prisonnier de Vincennes sous Louis XVI puis représentant du tiers état à la Révolution, vont nourrir une révolte qui s'ouvre à la fin du XVIII ${ }^{\mathrm{e}}$ siècle. Cet aristocrate, dont la jeunesse libertine est tout entière «[marquée] par des lettres de cachet $^{2} »$, fera en effet de ce système le symbole de l'arbitraire. Condamné à mort par contumace pour rapt et adultère, il est emprisonné à la demande de son père. Ses trois années de détention (mai 1777-décembre 1780) sont néanmoins l'occasion de nouer une correspondance avec son amante, Sophie de Monnier, enceinte et recluse dans un couvent.

La lettre écrite en prison constitue alors aussi bien le lieu d'un épanchement pathétique qu'un outil de contestation de l'autorité. Cette correspondance met en scène le caractère incommensurable du pouvoir institutionnel, et par là même les stratégies d'écrasement de l'individu captif. La peinture que le comte dresse de ses modalités d'incarcération fait de son expérience une torture physique et psychologique: les conditions matérielles de détention, et particulièrement le

1. Diderot, Lettre à la princesse Dashkoff, 3 avril 1771, Correspondance, Paris, Minuit, 1964, t. XI, p. 20.

2. Mirabeau, Lettre 18 du 29 sept. 1777 à Lenoir, Lettres écrites du donjon de Vincennes, (1777-1778), éd. de Béatrice Didier, Paris, Actes sud, 1998, p. 146. 
linge trop rare, la mauvaise qualité de la nourriture, de même que la vermine perpétuellement présente dans les cachots, avilissent le prisonnier. L'inconfort mais encore le secret absolu permettent aux autorités de manifester sans discontinuer leur emprise sur l'existence voire la survie du détenu. C'est ainsi que se révèle le pouvoir institutionnel de punir.

Il s'agit ainsi de comprendre dans quelle mesure l'écriture en prison participe à la dénonciation de l'institution carcérale : comment en effet Mirabeau présente-t-il les modes de contrôle institutionnel qui s'exercent à Vincennes? Quelles sont les stratégies du prisonnier pour échapper à la surveillance pénitentiaire?

Nous considèrerons par conséquent la lettre comme support d'une stratégie de lutte contre l'oppression: malgré la vigilance des autorités, le courrier permet la préservation d'un espace de liberté et la dénonciation d'une «institution totale ${ }^{3} »$ arbitraire.

Cette correspondance témoigne tout d'abord du contrôle exercé sur les détenus à Vincennes: privé de toute communication libre avec l'extérieur, Mirabeau présente assurément les effets dévastateurs de la surveillance. Ensuite, les contraintes carcérales s'exercent tout particulièrement sur le courrier, dévoilant ainsi l'avarice et même l'omniscience de l'institution. Enfin, les stratégies épistolaires de contournement de la règle font la preuve d'une résistance de l'écrivain.

\section{Mirabeau sous le sceau du secret}

À Vincennes, Mirabeau est presque absolument coupé du dehors et de sa vie passée: l'architecture écrasante du donjon, de même que le système de surveillance, privent le détenu de toute communication libre avec ses proches. La prison, pour l'ensemble de ces détenus, se fait sépulcre: la privation de liberté et de communication avec l'extérieur réduit le citoyen au néant. Mirabeau résume les procédés de mortification destinés à exclure le prisonnier de la vie civile, l'anaphore soulignant avec force les douleurs du manque: «Nulle correspondance, nulle société, nul éclaircissement de son sort, nulle distraction

3. Erving Goffman, Asiles. Études sur la condition sociale des malades mentaux et autres reclus, Paris, Minuit, 1968, p. 45-46. 
au présent, nulle connaissance de l'avenir... quelle effroyable mutilation de l'existence ${ }^{4}$ ! C Cette exclusion confine alors à la « mort civile ${ }^{5} »$.

\section{A. Une architecture de l'isolement}

L'enfermement chez Mirabeau, à l'instar de tout individu soumis aux pouvoirs de l'institution totale, fait particulièrement rupture dans une vie saturée de plaisirs. Ce contraste apparaît dans l'isolement concret et inexpugnable dressé par l'architecture carcérale entre l'intérieur et l'extérieur. En proie à l'insalubrité et au manque de confort, le prisonnier est ainsi totalement oppressé et mutilé, non seulement par les règles qui lui sont imposées, mais aussi par l'espace carcéral lui-même.

L'épaisseur des murs et des portes du donjon de Vincennes dresse inévitablement une barrière infranchissable mais symbolise encore la fermeté du pouvoir institutionnel. C'est pourquoi Mirabeau en décrit les moindres détails:

Il faudrait du canon de batterie et du plus gros calibre pour y faire brèche. Des fossés profonds d'environ quarante pieds, larges de vingt pas, et revêtus en pierres de taille, l'entourent. Ce revêtement est à pic, et vers le haut, il règne une corniche ou plutôt un talus qui saille tellement en dedans, qu'il faudrait se renverser pour le franchir, de sorte qu'un homme, parvenu dans les fossés, et sans intelligence au dehors, serait aussi sûrement renfermé que dans les tours.

Suit une enceinte, formée d'une seule entrée, que défendent deux sentinelles et trois portes ${ }^{6}$.

Cette description, qui s'organise de l'extérieur vers l'intérieur de la prison, suivant ainsi le parcours d'un futur détenu, souligne la solidité du bâtiment, en apparence inaltérable. Cette caractéristique architecturale, digne d'une forteresse médiévale, est à l'image de l'incommensurable autorité qui a incarcéré Mirabeau: l'accumulation de murs épais, de portes et de gardes paraît rendre toute invasion ou évasion apparemment vaine. Dans cette représentation, où une «corniche règne », l'édifice semble prendre le pouvoir au point de réifier l'indi-

4. Mirabeau, Lettre 18, 29 septembre 1777, à Lenoir, Lettres écrites du donjon, op. cit., p. 145 .

5. Ibid., p. $5^{8 .}$

6. Mirabeau, Des lettres de cachet et des prisons d'État, dans Euvres de Mirabeau, précédées d'une notice sur sa vie et ses ouvrages par Joseph Mérilhou, Paris, Lecointe et Pougin, 1835 [Hambourg, 1782], t. VII, p. 336-337. 
vidu. La coordination de «deux sentinelles et trois portes », à la limite du zeugme sémantique, place l'objet et l'humain sur un même plan et fait des professionnels de la surveillance des instruments au service de la machine institutionnelle. Il présente alors dans cet esprit les procédés qui visent à maintenir la sureté à Vincennes?. Si le bâtiment exclut la fuite d'un prisonnier, les surveillants eux-mêmes obéissent à des règles strictes. La routine qui prédomine dans ce système fait de ses occupants, condamnés ou surveillants, des automates soumis à l'institution: elle rythme leurs journées et chaque geste a pour objectif de sécuriser les lieux. De fait, cet isolement profond répond à une exigence institutionnelle aux dépens des êtres humains qui résident dans le château. Il est accentué par la relative privation de communication avec l'extérieur.

\section{B. Une condamnation au secret absolu}

Au donjon de Vincennes, les prisonniers sous lettre de cachet sont effectivement condamnés au secret absolu: «point de communications entre eux, pas de contacts avec l'extérieur, nulle promenade hors de la cellule, pas de livres autres que ceux fournis par le commandant du donjon; aucune liberté d'écrire, le papier était compté et devait être représenté après usage ${ }^{8}$ ».

Par conséquent, Mirabeau ignore le sort de ses proches ${ }^{9}$ et ne peut exercer ses fonctions d'époux et de père. D'ailleurs, ses deux enfants mourront en bas âge au cours de son incarcération ${ }^{10}$. Les captifs, à Vincennes, sont privés de tout rôle social et même de tout contact affectif, ce qui rend tout événement tragique plus cruel encore.

L'isolement devient alors un refrain de la correspondance de Mirabeau, comme il l'exprime dans une question rhétorique adressée à son père: «Vous m'avez condamné à une mort civile, ô mon père! et c'est beaucoup plus qu'à une mort violente [...]. Souffrir dans une

7. Mirabeau, Des lettres de cachet, op. cit., p. 337.

8. René de la Croix, duc de Castries, Mirabeau ou l'échec du destin, Paris, Librairie Arthème Fayard, 1960, p. 129.

9. Mirabeau, Lettre 1, juillet 1777, à Lenoir, Lettres écrites du donjon, op. cit., p. 31.

10. Mirabeau, Lettre 43, $1^{\text {er }}$ décembre 1778 et Lettre 69, 28 mai 1780, «Les amours qui finissent ne sont pas les nôtres ». Lettres à Sophie de Monnier. 1777-1780, éd. de Jean-Paul Desprat, Paris, Taillandier, 2010, p. 212 et 319-323. 
solitude profonde toutes les privations et toutes les inquiétudes, être arraché à tout ce qu'on aime, n'est-ce pas plus, infiniment plus que mouririll ?».

De surcroît, le détenu est condamné au mutisme. Dès l'entrée, on lui rappelle qu'il s'introduit dans la «maison du silence ${ }^{12}$ »; il se plaint alors, chaque fois qu'il est privé de la parole, de «cet affreux silence qui [l'] e enveloppé ${ }^{13} »$. Tout échange avec le personnel est proscrit lors des premiers temps de son incarcération. Par ailleurs, les porte-clefs ont ordre de taire tout ce qui concerne le sort du prisonnier.

La solitude et le silence, parce qu'ils le séparent des êtres aimés, constituent de ce fait un véritable tombeau. Mirabeau dénonce ainsi le trouble mental provoqué par le secret absolu et la claustration en exposant de ponctuels dédoublements terrifiants de lui-même : «je n’ai de compagnon, déclare-t-il, que ma tristesse et mes craintes ${ }^{14} »$. D’un point de vue rhétorique, cette allégorie doit créer un effet pathétique sur le destinataire; d'un point de vue psychologique, l'extrait annonce la dégradation mentale du détenu, qui déploie un mécanisme de défense face à un état de grande vulnérabilité. Dans les moments de désespoir, en particulier lorsqu'il imagine ne plus revoir son amante, le comte en vient effectivement à s'adresser à lui-même: «Agité par mille idées contraires, tantôt j'écoute en silence cette voix qui me parle, qui mappelle, qui me crie: Elle est perdue pour toi; voilà ta dernière demeure; tu ne la reverras plus; et je suis prêt à me frapper ${ }^{15}$. » Ce double agressif peuple son imagination et emplit le vide de sa cellule; incarnation de son accablement, il est engendré par la privation de communication avec quiconque.

Cette sensation de solitude est alors renforcée par l'absence de réponse aux lettres qui ont pu être envoyées ${ }^{16}$. La coupure absolue avec

11. Mirabeau, Mémoire adressé par Mirabeau à son père, décembre 1777, Lettres écrites du donjon, op. cit., p. 332.

12. Mirabeau, Des lettres de cachet, op. cit., p. 340.

13. Mirabeau, Lettre 3, août 1777, à Sophie, Lettres écrites du donjon, op. cit., p. $3^{8 .}$

14. Mirabeau, Lettre 13, septembre 1777, à Lenoir, Lettres écrites du donjon, op. cit., p. 107 .

15. Mirabeau, Lettre 15, samedi 13 septembre 1777, à Sophie, Lettres écrites du donjon, p. 128.

16. Mirabeau, Lettre 4, août 1777, à Sophie et Lettre 18, 29 septembre 1777, à Lenoir, Lettres écrites du donjon, op. cit., p. 51 et 143. 
l'extérieur donne finalement la sensation que plus rien n'existe que ce tombeau des vivants: "Je reçois ta lettre du 31, mon aimable amie, dans un instant où je croyais que le donjon de Vincennes survivait au reste du monde, et que toute la terre et ses habitants étaient engloutis ${ }^{17}$.

D’une façon générale, Mirabeau présente dans ses écrits carcéraux l'ensemble des dispositifs établis pour mieux contrôler le captif tels qu'ils sont notamment présentés par Michel Foucault. Pour maintenir la discipline, il s'agit en effet de s'assurer de la «clôture » de l'individu, mais aussi du «quadrillage» de l'espace ("à chaque individu sa place») en cellule ou geôle, d'une stricte régulation de son emploi du temps et d'une surveillance permanente ${ }^{18}$. Le caractère pathétique des méfaits psychologiques de l'incarcération relève alors chez Mirabeau d'une stratégie argumentative. La rhétorique du pathos dans cette correspondance est une façon d'émouvoir ses lecteurs et de les convaincre de l'iniquité de la prison, lorsqu'elle est appliquée sous lettre de cachet.

Mirabeau dénonce plus particulièrement le contrôle institutionnel exercé sur la correspondance du détenu et donc sur l'expression de ses sentiments.

\section{Un courrier sous surveillance : la gestion administrative, frein à la libre expression des émotions}

Le courrier, source d'apaisement pour le prisonnier, constitue l'un des rares modes de communication avec l'extérieur. Il s'agit d'un pont permettant au prisonnier de franchir l'obstacle des murs de Vincennes. Il est aussi l'occasion de manifester ses émois profonds et intérieurs. Toutefois, parce qu'elle le place sous surveillance, l'administration pénitentiaire maîtrise encore cet espace restreint de liberté, d'une part en limitant la distribution du matériel épistolaire, d'autre part en contrôlant le contenu des lettres.

\section{A. Une distribution parcimonieuse du matériel épistolaire}

Lorsque les prisonniers obtiennent l'autorisation de mener une correspondance, un nouvel obstacle s'offre à eux: la difficulté à obtenir le

17. Mirabeau, Lettre 66, 21 février 1780, «Les amours qui finissent», op. cit., p. 313 .

18. Voir Michel Foucault, Surveiller et punir. Naissance de la prison, Paris, Gallimard, 1975 . 
matériel nécessaire pour écrire. Non seulement le papier coûte cher au XVIII ${ }^{\mathrm{e}}$ siècle ${ }^{19}$, mais, à Vincennes, il est distribué avec parcimonie. Par conséquent, Mirabeau subit la menace de ce qu'il nomme la « disette ${ }^{20} »$, considérant ainsi le papier comme nécessaire à sa vie, au même titre que la nourriture. De fait, en septembre 1777, une lettre adressée à Sophie ne comporte pas les traditionnelles salutations par manque de place: «Le papier me manque, ma Sophie ${ }^{21}$ », déplore le signataire à la fin de son courrier. Il en est de même de l'encre. C'est pourquoi Sophie emploie les méthodes les plus singulières pour écrire à son amant: elle rédige ses missives sur « des bouts de papier et tente vainement d'écrire avec du café, puis, avec une espèce d'encre, obtenue en faisant macérer des clous rouillés dans du vinaigre ${ }^{22} »$. De même, Mirabeau, ne vivant parfois que de "pillage ${ }^{23}$ ", rédige sur des pages arrachées dans des livres, voire sur des images et sur des cartes ${ }^{24}$. Tout est bon pour maintenir cette correspondance essentielle à leur cœur.

L'avarice de l'institution en matière de papier contraint donc les correspondants à écrire des lettres brèves et produit ainsi une forme de frustration ${ }^{25}$. Les correspondants captifs doivent réduire autant que possible les marges et la taille de la graphie. Ainsi, Mirabeau mesure de façon obsessionnelle l'amour de Sophie à la longueur de la lettre ainsi qu'à l'effort de chacun pour écrire beaucoup sur peu de papier ${ }^{26}$. S'évertuant à allonger son propos, il reproche sans cesse à Sophie son manque d'économie; l'impératif, les points de suspension et les froids appellatifs marquent sa contrariété : «écris-les plus fins, et n’y mets pas

19. Mirabeau, Lettre 11, 27 août 1777, à Sophie, Lettres écrites du donjon, op. cit., p. 100.

20. Mirabeau, Lettre 3, août 1777, à Sophie, Lettres écrites du donjon, op. cit., p. 44 .

21. Mirabeau, Lettre 17, 19 septembre 1777, à Sophie, Lettres écrites du donjon, op. cit., p. 142.

22. René de la Croix, op. cit., p. 128.

23. Mirabeau, Lettre 3, août 1777, à Sophie, Lettres écrites du donjon, op. cit., p. 44 .

24. Béatrice Didier, notes sur le texte, dans Lettres écrites du donjon, op. cit., p. 315 .

25. Mirabeau, Lettre 24, 10 novembre 1777, à Lenoir, Lettres écrites du donjon, op. cit., p. 189 .

26. Mirabeau, Lettre 23, $1^{\text {er }}$ novembre 1777, à Sophie, Lettres écrites du donjon, op. cit., p. 180 . 
tant de $\operatorname{marge}^{27}$ »; «Les lignes sont si écartées, les mots si larges, que rien au monde n'est si hypocrite que ton écriture ${ }^{28}$ ». La graphie devient alors une métonymie de l'amante elle-même; graphologue avant l'heure, Mirabeau perçoit dans les signes apposés par sa bien-aimée une disposition d'âme: "Sophie, chacune de mes pages contient environ 72 lignes, chaque ligne environ 25 à 30 mots; chacune de tes pages porte 40 lignes, et chacune de tes lignes environ 14 mots. Compare, et rougis. Tu m’as écrit 2,240 mots en 80 jours; c'est 28 mots par jour. Quel effort! aussi tes yeux sont fatigués. Ah! Sophie, plus de silence de 80 jours $^{29}$. » D'une certaine manière, il reproduit avec son amante la froide rigueur que lui inflige l'institution; ses injonctions et son obsession tatillonne sont vraisemblablement induites par le système.

\section{B. Le contrôle du contenu des lettres: précautions épistolaires et autocensure du captif}

En outre, la vigilance continue exercée à Vincennes s'applique encore au contenu de la correspondance. Cacheter une lettre demeure impossible car «ce n'est pas la règle», répond-on à Mirabeau ${ }^{30}$. Tout échange épistolaire doit obtenir permission et doit s'adapter aux exigences de la hiérarchie dans la mesure où le lieutenant de police Lenoir a accès à l'ensemble des écrits ${ }^{31}$. De cette façon, la lettre écrite en prison comporte au moins deux destinataires : le correspondant initial mais encore le lieutenant général de police. Ce contexte particulier modifie ainsi les modes d'écriture et de lecture du document: le contenu est soumis à la domination de l'autorité carcérale. L'intimité du prisonnier, plus particulièrement ses échanges amoureux, sont ainsi profanés au profit de la sûreté carcérale.

Ainsi, le comte est contraint de demander l'autorisation d'écrire et de soumettre le contenu de ses lettres. Ces demandes se font supplications lorsqu'il s'agit d'écrire aux proches, notamment à sa femme: «[ma lettre à mon amie] vous apprendra, mieux que je pourrais l'expri-

27. Mirabeau, Lettre 2, 16 décembre 1778, de Sophie, Lettres écrites du donjon, op. cit., p. 328 .

28. Mirabeau, Lettre 39, s. d., «Les amours qui finissent», op. cit., p. 188.

29. Mirabeau, Lettre 41, s. d., «Les amours qui finissent», op. cit., p. 204.

30. Mirabeau, Des lettres de cachet, op. cit., p. 331.

31. Mirabeau, Lettre 21, 17 octobre 1777, au maréchal duc de Noailles, Lettres écrites du donjon, op. cit., p. 170. 
mer, le bien que vous m’avez fait, et la gratitude dont je suis pénétré... Je vous supplie qu'elle lui soit remise ${ }^{32} »$. La potentielle censure du courrier enjoint Mirabeau à se faire circonspect: «Monsieur, je vous conjure de permettre que celle que je vous adresse tombe dans ses mains; ou [s'il] s'y trouve quelque indiscrétion, daignez faire raturer tout ce qui vous déplaira. [...] Je suis prêt à tout écrire sous votre dictée ${ }^{33}$. $\gg$ Ces conditions induisent une forme de déférence voire de flatterie à l'égard des autorités. Tout d'abord, selon la formule consacrée, Mirabeau prend congé de Lenoir avec un grand respect ( «Jai l'honneur d'être, avec des sentiments respectueux, monsieur, votre très humble et très obéissant serviteur $\left.{ }^{34} »\right)$. Mais ces égards sont encore perceptibles lorsque Mirabeau écrit à Sophie une lettre qu'il sait lue par le lieutenant: «ne comprends-tu pas [que] je ne risque point de mettre ici des choses qui déplaisent à notre bienfaiteur?... ${ }^{35}$ » L'intensité de la gratitude fait soupçonner une stratégie de Mirabeau pour mieux permettre la circulation de ses lettres. Ce contexte exige encore une forme d'autocensure: «Les réflexions naissent en foule; mais je les réprime $^{36}$ », déclare-t-il à Lenoir ; "Chère amie, annonce-t-il à Sophie, si quelques expressions de ma dernière lettre t'ont paru ambiguës, c'est que j'avais des raisons de craindre que le moindre défaut de circonspection ne t'en privât ${ }^{37}$.» L'épistolier déplore alors les contraintes imposées, ses lettres devenant un «petit nombre de lignes dictées par l'amour, mais par l'amour enchaîné par la prudence ${ }^{38} »$. Le lieutenant Lenoir impose effectivement des règles qui nuisent à la liberté d'échange. Par exemple, il exige que Mirabeau vouvoie son amante ${ }^{39}$.

32. Mirabeau, Lettre 47, 5 avril 1778, à Lenoir, Lettres écrites du donjon, op. cit., p. 292.

33. Mirabeau, Lettre 40, 2 mars 1778, à Lenoir, Lettres écrites du donjon, op. cit., p. 248.

34. Mirabeau, Lettre 18, 29 septembre 1777, à Lenoir, Lettres écrites du donjon, op. cit., p. 155 .

35. Mirabeau, Lettre 43, 20 mars 1778, à Sophie, Lettres écrites du donjon, op. cit., p. 269.

36. Mirabeau, Lettre 37, 8 février 1778, à Lenoir, Lettres écrites du donjon, op. cit., p. 238.

37. Mirabeau, Lettre 43, 20 mars 1778, à Sophie, Lettres écrites du donjon, op. cit., p. $263-264$.

38. Mirabeau, Lettre 43, 20 mars 1778, à Sophie, Lettres écrites du donjon, op. cit., p. 270.

39. Mirabeau, Lettre 35, 14 janvier 1778, à Lenoir, Lettres écrites du donjon, op. cit., p. 229-230. 
L'expression épistolaire des émotions du captif est donc elle aussi sous surveillance. Cette écriture contrainte s'oppose tout à fait à l'authenticité attendue et/ou supposée du genre de la lettre. D'une façon générale, la prison non seulement impose une barrière entre l'intérieur et l'extérieur mais viole aussi l'intimité du captif. La prolifération des regards posés sur le détenu, en particulier sur sa correspondance la plus personnelle, modifie son écriture.

Toutefois, Mirabeau n’a en vérité pas cédé sous le poids écrasant de l'institution. Il trouve dans l'écriture le remède nécessaire aux souffrances carcérales. À l'opposé du figement imposé à Vincennes, le reclus rédigeant des lettres peut se projeter dans un ailleurs et simuler des échanges véritables. Même sans être cachetée, la lettre permet de se garantir des effets dévastateurs de la prison.

\section{Stratégies épistolaires de contournement de la règle carcérale}

L'échange de lettres d'amour constitue un véritable répit à la souffrance carcérale, la métaphore du remède devenant un leitmotiv des lettres adressées à Sophie ${ }^{40}$. À cette métaphore traditionnelle s'ajoute une image dont la résonance en prison est plus profonde encore: la lettre du détenu à son amante captive offre une évasion et une protection imaginaire; elle est l'occasion de percer l'obstacle carcéral et de bâtir un édifice qui préserve l'intimité des amoureux : «Souffrez [...] que nos soupirs franchissent la distance des lieux et l'épaisseur des murs $^{41}$; « «e n'ai qu'un asile, que le despotisme qui me foudroie ne peut atteindre, et dont il ne pourra m’arracher [ton amour] ${ }^{42}$ ", déclaret-il à Sophie. Cette métaphore résume les bienfaits, même illusoires, de l'écriture d'une correspondance amoureuse en prison.

Pour mieux préserver leur intimité amoureuse, Gabriel et Sophie imaginent alors des stratégies de contournement de la règle carcérale: ils organisent des échanges épistolaires clandestins et créent des messages

40. Mirabeau, Lettre 28, 28 décembre 1777, à Sophie, Lettres écrites du donjon, op. cit., p. 204.

41. Mirabeau, Lettre 44, 29 mars 1778, à Sophie, Lettres écrites du donjon, op. cit., p. 272. Notons l'usage d'un «vous» adressé à Sophie.

42. Mirabeau, Lettre 15, 13 septembre 1777, à Sophie, Lettres écrites du donjon, op. cit., p. 127. 
cryptés. Ils fondent ainsi plus profondément encore l'édifice amoureux qui les protège de la surveillance permanente.

\section{A. Une correspondance clandestine}

Pour mieux se préserver du réseau de surveillance, ils commencent par s'attacher la complicité de membres de l'institution qui vont leur transmettre des lettres clandestines. Le lieutenant de police Lenoir, tout d'abord, communique des lettres à Sophie, mais exige que l'original lui revienne. Cette sollicitude s'explique peut-être par le fait que Lenoir et Mirabeau sont tous deux francs-maçons et parce que le lieutenant «abhorrait les physiocrates» dont le père de Mirabeau est l'un des représentants. Cette méfiance de Lenoir à l'égard de «l'Ami des hommes » bénéficie au comte captif puisqu'il l'autorise notamment à correspondre avec sa maîtresse par l'intermédiaire de Boucher, premier commis du Secret. Les hautes fonctions de ce dernier ne lui permettant pas de se charger des lettres, il le demande au policier Brugnières, qui l'avait arrêté à Amsterdam et devint néanmoins son facteur clandestin ${ }^{43}$.

Il s'adresse également au chirurgien Fontelliau, qui lui rend visite à Vincennes. Ce dernier lui donnera des nouvelles de sa mère, qu'il soigne au Val d'Osne, et transmettra des lettres de Mirabeau à Sophie ${ }^{44}$. L'incommodité devient ainsi un atout pour le prisonnier. Toutefois, ce complice le trahira lorsqu'il se sentira en danger, comme en témoigne la lettre du détenu à Rougemont datant de septembre $177^{45}$.

Il s'agit également de corrompre les gardes afin d'obtenir des faveurs, car, selon Mirabeau, "passer des lettres ici [...] ne se peut guère qu'à force d'argent». Les amants échangent alors sur les stratégies envisageables. Sophie songe à faire passer les lettres par les fenêtres; Mirabeau pense à placer son courrier sous un banc dans le jardin où les prisonniers se promènent afin qu'une sentinelle puisse discrètement le récupérer ${ }^{46}$. Nous ne savons pas si ces techniques ont été adoptées mais, d’après Béatrice Didier, «il y aurait eu aussi des

43. René de la Croix, op. cit., p. 130-131.

44. Voir par exemple Mirabeau, Lettre 15, 13 septembre 1777, à Sophie, Lettres écrites du donjon, op. cit., p. 126-127.

45. Mirabeau, Lettre LXV, à M. de Rougemont, 20 septembre 1778, dans Euvres de Mirabeau, op. cit., t. V, p. 103-113.

46. Mirabeau, Lettre 7, août 1777, à Sophie, Lettres écrites du donjon, op. cit., p. $72-73$. 
lettres secrètes à Sophie plus suggestives encore, qui ne passaient pas par Lenoir et auraient été perdues ${ }^{47}$ ». Cette stratégie n’est en rien originale. Comme l'expose Mirabeau, d'autres illustres prisonniers ont soudoyé leurs porte-clefs pour pouvoir correspondre clandestinement, et même pour s'évader ${ }^{48}$.

Plusieurs membres de l'institution œuvrent donc en faveur du reclus. En résumé, il existe trois types de lettres adressées à Sophie : les lettres officielles (autorisées et non cachetées), les lettres clandestines (distribuées par des membres haut placés de l'institution à l'insu de Rougemont) et les lettres illicites (passant par des complices rémunérés ou des connaissances privées, à l'insu de Lenoir et Rougemont). Une publication de cette correspondance indiquant ces différents niveaux, à la manière par exemple des strates indiquées dans les Essais de Montaigne à l'aide de barres obliques (I, //, //I) ou de lettres (A, B, C), pourrait éventuellement être pertinente; elle pourrait ainsi mettre en valeur les probables variations du discours.

\section{B. Des messages cryptés}

Afin de prolonger la dissimulation du contenu épistolaire, le message peut être crypté à l'aide de la mauvaise qualité de la graphie : "Tu sais que je me suis félicité quelquefois d'avoir si mal écrit ce que je t’adressais, que d'autres n'ont pas pu le déchiffrer ${ }^{49}$. . Le substantif «griffonnage» revêt ainsi une connotation positive ${ }^{50}$. La reconnaissance des caractères de l'être aimé dit l'intimité ancienne des corps et préserve de tout regard voyeur ou censeur. Les amants utilisent encore de l'encre sympathique faite à base de citron $^{51}$ pour placer, dans «les contenus montrables $[. .$.$] des interpolations secrètes { }^{52}$ ».

47. Béatrice Didier, «Préface. Un an dans la vie de Mirabeau», dans Lettres écrites du donjon, op. cit., p. 17.

48. Mirabeau, Des lettres de cachet, op. cit., p. 367-368. Voir Cardinal de Retz, Mémoires, éd. de Simone Bertière, Paris, Classiques Garnier, 2010, p. 1101-1102, et Marguerite-Jeanne de Staal-Delaunay, Mémoires, dans Catriona Seth (éd.), La fabrique de l'intime. Mémoires et journaux de femmes du XVIII siècle, Paris, Robert Laffont, 2013, p. 202.

49. Mirabeau, Lettre 2, 16 décembre 1778, de Sophie, Lettres écrites du donjon, op. cit., p. 331.

5o. Mirabeau, Lettre 45, s. d., «Les amours qui finissent», op. cit., p. 223.

51. Mirabeau, Lettre 45, s. d., «Les amours qui finissent», op. cit., p. 232.

52. Michel Delon, «Lettres d'amour, lettres de combat», Revue de la BnF, 35, 2, 2010 [en ligne]. 
Enfin, les amants s'inventent un langage codé. D’une façon générale, cette pratique est fréquente dès la naissance d'un service postal. En effet, Voltaire, dans le Dictionnaire philosophique, souligne l'absence de sécurité et de confidentialité du courrier qui passe par le système postal de son temps: "Jamais le ministère qui a eu le département des postes n'a ouvert les lettres d'aucun particulier, excepté quand il a eu besoin de savoir ce qu'elles contenaient ${ }^{53}$.» Au XVIII ${ }^{\mathrm{e}}$ siècle, la surveillance royale permanente suppose que l'on préserve la confidentialité par plusieurs stratagèmes, en particulier un code convenu au préalable entre les correspondants. D'ailleurs, d'après Geneviève Haroche-Bouzinac, cette «utilisation du langage chiffré ou codé, [demeure] sans doute suffisamment fréquente pour avoir motivé une interdiction de François $1^{\text {er }}$ en $1546^{54}$ ». Cette stratégie devient essentielle en prison, dans laquelle la surveillance est accrue. Gabriel et Sophie inventent notamment un code secret pour les noms propres et cumulent les stratégies de création de surnoms, telles qu'elles ont été décrites par Diane Beelen Woody ${ }^{55}$, en particulier pour ceux qui désignent leurs complices. Par exemple, Gabriel se fait appeler «Gaub » et Sophie «Sélo». Par exemple encore, le policier Brugnières servit Mirabeau avec tant de dévouement que celui-ci le nomma Pylade ${ }^{56}$, à l'instar du dévoué ami d'Oreste, fils et assassin de Clytemnestre. Dans les lettres des amants, seule l'initiale de ce surnom apparaît: «P... ${ }^{57}$. Pour leur complice, ils emploient ainsi une double technique de dissimulation du nom : le surnom et l'usage de son initiale. Ces précautions extrêmes préservent non seulement la communication entre les amants mais encore Boucher et Lenoir, qui pourraient ainsi se dédouaner auprès de l'institution.

De surcroît, ils créent des signes hiéroglyphiques et un alphabet cryptographique. Ainsi, le symbole du cœur peut signifier «nous» et tous les dérivés de la première personne du pluriel; un « $\mathrm{A}$ » majuscule peut signifier «Je»... Enfin, ils imaginent un langage chiffré, qui, pour p. 46.

53. Cité par Geneviève Haroche-Bouzinac, L'épistolaire, Paris, Hachette, 1995,

54. Ibid., p. 47 .

55. Voir, dans le présent volume, Diane Beleen Woody, «Surnoms et écriture codée dans les lettres de Mme de Graffigny».

56. René de la Croix, op. cit., p. 132.

57. Mirabeau, Lettre 4, août 1777, à Sophie, Lettres écrites du donjon, op. cit., p. 50 et suivantes. 
être percé, a nécessité l'attention de Paul Cottin, bibliothécaire de l'Arsenal, et les services d'un officier du renseignement ${ }^{58}$. Cette pratique est adoptée par le marquis de Sade, obsédé par les signaux supposés indiquer la date de sa libération, mais aussi le cardinal de Retz, qui a composé un code qu'il nomme «indéchiffrable» pour mener à bien son évasion ${ }^{59}$.

La survie du prisonnier, assurée par l'échange de lettres, s'appuie sur des stratégies épistolaires visant à masquer le contenu des propos. Ces procédés sont des motifs topiques de la littérature amoureuse. Ovide, dans L'art d'aimer, conseillait déjà aux amants d'«éluder la surveillance» d'Argus, le chien aux cent yeux chargé par Junon de veiller sur Io. Il recommandait aux femmes de crypter le message: «que la même main s'accoutume à varier son écriture de plusieurs manières $[. .$.$] , et il n'est pas prudent de répondre [sur les mêmes$ tablettes] avant d'avoir bien gratté la cire, pour qu'elles ne gardent pas trace d'une double écriture. Lorsque vous écrivez à votre amant, ayez toujours l'air de vous adresser à une femme; dans vos billets, dites « elle» où il faut «il » »; il préconisait le recours à une complice dissimulant la lettre sous son corsage ou «offrant son dos et portant le message écrit sur sa peau » et prônait la corruption des gardiens; enfin il proposait l'emploi de lait frais, «moyen assuré de tromper les yeux », car «il suffit, pour lire les caractères, de le saupoudrer de charbon pulvérisé60 ${ }^{60}$. Toutefois, ces motifs sont revivifiés par le contexte d'écriture amoureuse que connaissent Gabriel et Sophie. Leur correspondance, finalement assez peu censurée dans la mesure où Lenoir est complice, permet encore de s'épancher avec sincérité et de prolonger l'échange amoureux, qui se transforme en ébats fantasmés. Il s'agit donc de bâtir un édifice imaginaire propre à préserver les amants captifs et de compenser la privation d'intimité et la frustration générées par la prison par l'expression sincère de leur amour.

D’une manière générale, ce n’est donc pas véritablement l'amour pour Sophie qui préserve son amant mais plutôt l'écriture de cet

58. Voir Paul Cottin, «Introduction", Sophie de Monnier et Mirabeau d'après leur correspondance secrète inédite (1775-1789), Paris, Plon, 1903, p. LXV-LXX.

59. Voir Guillaume Patin, «Les secrets commerces de Sade», Dix-huitième siècle, 41, 2009, p. 655-667, et Cardinal de Retz, op. cit., p. 1127-1128.

6o. Ovide, L'art d'aimer, éd. et trad. d'Henri Bornecque, Paris, Les Belles Lettres, 1924 , L. III, p. 78 , v. 483-498; p. 83-84, v. 651-658; p. 82, v. 619-632. 
amour. Écrire, qu'il s'agisse de lettres officielles, clandestines ou illicites, est une façon de vivifier un esprit engourdi par l'immobilité contrainte et de lutter contre la surveillance permanente qui règne à Vincennes.

\section{Conclusion}

Somme toute, l'écriture en prison préserve l'individu et l'amant mais nourrit encore l'homme politique. Si Mirabeau a été enfermé pour rapt et adultère, il n'alimente pas moins sa relation avec Sophie de Monnier, mettant ainsi de nouveau en question les décisions institutionnelles. La correspondance, dans laquelle il ne freine en aucun cas des sentiments qui l'ont conduit à sa perte, offre un rempart qui préserve son intimité et libère son énergie vitale.

Bien que soumis au pouvoir institutionnel, Mirabeau s'insurge par écrit contre «une injuste prison ${ }^{61}$ ", forme d'hypallage visant à la fois son père et le système en général, et contre l'arbitraire des lettres de cachet. En raison de l'obligation absolue du secret maintenu à Vincennes, il adopte la seule stratégie envisageable, l'écriture épistolaire. La correspondance en prison demeure l'unique moyen de défendre sa cause dans la mesure où aucun procès n'a lieu. Dans ces circonstances, le comte tire parti de la double lecture de son courrier ${ }^{62}$. Il sait ainsi jouer de la multiplication des lecteurs et faire résonner autant que possible ce qui lui paraît n'être que justice.

Les lettres de Mirabeau offrent à leur auteur captif des moments de répit et d'évasion qui lui ont permis de résister aux pires conséquences de l'incarcération. La rédaction et la transmission de sa correspondance lui permettent également de retourner l'arme institutionnelle contre elle-même: il développe cette forme épistolaire de révolte contre la surveillance absolue pour dénoncer l'arbitraire et les abus, notamment judiciaires. Représentatif en cela d'une pensée éclairée, Mirabeau souhaite se libérer de toute forme d'asservissement, qu'il soit matériel ou intellectuel et participera par conséquent à l'abolition des lettres de cachet. Inspiré par la satire 3 d'Horace, pilier de la culture

61. Mirabeau, Lettre 2, $1^{\text {er }}$ août 1777, à Lenoir, Lettres écrites du donjon, op. cit., p. 33.

62. Mirabeau, Lettre 48, 13 avril 1778, à Lenoir, Lettres écrites du donjon, op. cit., p. 294. 
humaniste ${ }^{63}$, il déclare effectivement aspirer à un bel avenir «sans [se] rendre esclave $[. .$.$] de personne, ni d'aucun préjugé$ e $^{4}$ », car, expose-t-il à Sophie, «c'est lorsque l'esprit de l'esclavage est assez enraciné [...] que l'on regarde de bonne foi comme des fous ceux qui lui résistent et affichent d'autres principes ${ }^{65}{ }$.

63. «Huc propius me / Dum doceo insanine omnes vos ordine adite » « Lorsque je vous apprends que vous êtes tous fous, je suis content de marcher à votre tête. Suivez-moi », traduction de Giacomo Casanova, Sur les odes d'Horace, dans Histoire de ma vie, éd. de Francis Lacassin, Paris, Robert Laffont, 1993, t. I, p. 1419.

64. Mirabeau, Lettre 3, 9 août 1777, à Sophie, Lettres écrites du donjon, op. cit., p. 46.

65. Mirabeau, Des lettres de cachet, op. cit., p. 379. 